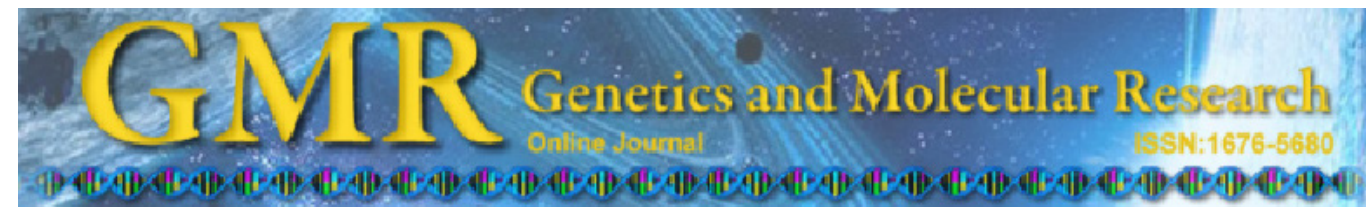

\title{
Bibliometric network analysis of glaucoma
}

\author{
L.-Y. Dong ${ }^{1 *}$, M. Yin ${ }^{2 *}$ and X.-L. Kang ${ }^{1}$ \\ ${ }^{1}$ Department of Ophthalmology, \\ Xinhua Hospital Affiliated to Shanghai Jiao Tong University School of Medicine, \\ Shanghai, China \\ ${ }^{2}$ Department of Pediatric Cardiothoracic Surgery, \\ Shanghai JiaoTong University Affiliated Shanghai Children's Medical Center, \\ Shanghai, China \\ *These authors contributed equally to this study. \\ Corresponding author: X.-L. Kang \\ E-mail: xli.kang@gmail.com
}

Genet. Mol. Res. 13 (2): 3577-3585 (2014)

Received August 29, 2012

Accepted July 26, 2013

Published May 9, 2014

DOI http://dx.doi.org/10.4238/2014.May.9.1

\begin{abstract}
Elevated intraocular pressure is recognized as the principal risk factor for development of optic nerve head $(\mathrm{ONH})$ injury. Lamina cribrosa (LC) cells and astrocytes are two types of cells in the $\mathrm{ONH}$. We attempted to identify more target genes and predict their underlying molecular mechanisms. In this study, we performed meta-analysis of the data from two microarray sets containing samples from LC cells and astrocytes each. Our analysis indicated that 47 differentially expressed genes (DEGs) had been identified, and 24 of them were used to construct a bibliometric network with other related genes, including GSTT1 ENO2, CPE, PTN, PTGDS, IL6, MMP1, and EGFR. Further, our results predicted these genes might be involved in glaucoma development through Toll-like receptor signaling pathway, ErbB signaling pathway, and glioma and other cancer-related pathways. Therefore our study provides potential target genes and pathways for future therapeutic studies of glaucoma.
\end{abstract}

Key words: LC cells; Target genes; Bibliometric network; Glaucoma; Meta-analysis 


\section{INTRODUCTION}

Glaucoma is a form of degenerative optic neuropathy characterized by optic nerve axonal degeneration, optic nerve head (ONH) excavation, and visual field defects (Wein and Levin, 2002; Naka et al., 2010). It is the second leading cause of blindness worldwide, affecting approximately 70 million people (Quigley and Broman, 2006). Glaucoma is often associated with elevated intraocular pressure (IOP). Elevated IOP can induce changes in the $\mathrm{ONH}$ at the lamina cribrosa (LC) region, including activation of astrocytes, remodeling of the extracellular matrix (ECM), as well as inhibition of retrograde transport of neurotrophins to retinal ganglion cells (RGCs).

Reactive astrocytes increase the expression of various cell surface molecules that play important roles in cell-cell recognition and in cellular adhesion to substrates, as well as that of various growth factors, cytokines, and receptors involved in glaucoma. For example, reactive astrocytes could express several ECM remodeling proteins such as elastin (Hernandez, 1992; Pena et al., 2001), tenascin, and metalloproteinases (Yan et al., 2000; Agapova et al., 2003). Increased synthesis and deposition of ECM proteins in the LC region may disrupt nutritional and mechanical support to RGC axons, resulting in RGC atrophy. Interestingly, several studies have shown that the transition of quiescent astrocytes into the reactive phenotype needs to be induced by the expression of transforming growth factor (TGF)- $\alpha$ and TGF- $\beta$, ciliary neurotrophic factor (CNTF), fibroblast growth factor 2, platelet-derived growth factor (PDGF), and their receptors, which, in turn, alters the expression of ECM protein (Fuchshofer, 2011). TGF- $\beta 2$ increases the expression of elastin, collagen VI $\alpha-2$ chain (ColVI $\alpha 2$ ), collagen VI $\alpha-3$ chain (ColVI $\alpha 3)$, matrix metalloproteinases (MMP)-2, tissue inhibitor of metalloproteinase (TIMP)-1/-3, and plasminogen activator inhibitor (PAI-1) in the astrocytes of glaucoma patients (Neumann et al., 2008). Treatment of ONH astrocytes and LC cells with exogenous TGF- $\beta 2$ causes an increase in the synthesis and secretion of ECM protein through canonical Smad signaling pathway. Knockdown of either Smad2 or Smad3 via small interfering RNA reduces TGF- $\beta 2$-stimulated ECM proteins in ONH astrocytes and LC cells (Zode et al., 2009, 2011). In glaucoma, reactive astrocytes could migrate from the cribriform plates into the nerve bundles and synthesize neurotoxic mediators such as nitric oxide (NO) and tumor necrosis factor (TNF- $\alpha$ ), which may be released near the axons causing neuronal damage (Miao et al., 2010). Obstruction of anterograde and retrograde axonal transport at the $\mathrm{ONH}$ is also one of the initiators of the survival and death mechanisms that affect both RGC axons and cell bodies. Brain derived neurotrophic factor (BDNF) moves from the brain to the RGCs on the trkB receptor, and its retrograde transport is obstructed in acute and chronic glaucoma models (Iwabe et al., 2007). Overexpression of BDNF delays RGC death in experimental glaucoma (Chen and Weber, 2001). CNTF is also expressed in cells of all retinal layers and in the retinal pigment epithelium as well as in the ONH. Virally mediated overexpression of CNTF exerts a protective effect over RGC after optic nerve injury (Pease et al., 2009).

However, the mechanism of ONH injury in glaucoma remains obscure, and more potential genes are necessary to be mined. Microarray technology has recently been applied to glaucoma models, demonstrating the upregulation and downregulation of many genes associated with $\mathrm{ONH}$ injury (Kompass et al., 2008; Kirwan et al., 2009). Meta-analysis provides a powerful tool for analyzing microarray experiments by combining data from multiple studies; it also presents unique computational challenges. Therefore, we attempted to further measure the transcription profiles of LC cells and astrocytes by meta-analysis method and predict their underlying molecular pathways. These underlying genes and pathways are potential targets for future studies of glaucoma. 


\section{MATERIAL AND METHODS}

Meta-analysis is a suitable framework to conduct an objective, integrative, and comparative study of multiple related microarray gene expression profiles and analysis of differentially expressed genes (DEGs). Two glaucoma-related expression profiles GSE2378 and GSE13534 were obtained from Gene Expression Omnibus (GEO) (http://www.ncbi.nlm.nih. gov/geo/), which are based on the Affymetrix Human Genome U95 Version 2 Array and Affymetrix Human Genome U133A Array, respectively.

In the GSE13534 dataset, the LC cell lines were generated from human ONH explants as described previously and supplied by Alcon Research Labs at Fort Worth, TX (Lambert et al., 2001). The normal LC cell lines were obtained from donors with no history of eye disease and primary open-angle glaucoma (POAG) LC cell lines were obtained from donors with a documented history of POAG (Kirwan et al., 2009). Two POAG samples and two controls were used for analysis. In the GSE2378 dataset, expression profiling was performed for the astrocytes obtained from the $\mathrm{ONH}$ of patients with glaucoma. The $\mathrm{ONH}$ is the site of damage in glaucomatous optic neuropathy. Studies suggest that damage in glaucoma is mediated by reactive astrocytes (Hernandez et al., 2002). Seven glaucoma samples and six normal donors were used for analysis. Together, a total of nine glaucoma samples and eight controls were selected to identify DEGs in this study.

The microarray files were analyzed using the open source statistical package $\mathrm{R}$ version 2.12.1 (http://www.r-project.org/) and Bioconductor (Gentleman et al., 2004). For the two datasets, the RankProd package (Hong et al., 2006) and limma package (Diboun et al., 2006) were used to identify the DEGs. The DEGs with a percentage of false-positives (PFP) (Hong et al., 2006) of $<0.05$ and a fold change of $>2$ were considered differentially expressed between the samples and controls.

For showing the potential connection between DEGs, the bibliomics network was constructed. Biblio-MetReS creates networks that can be compared to those of other user-friendly tools. Furthermore, analysis of full text documents provides more complete reconstructions than those that result from using only the abstract of the document (Usie et al., 2011).

First, register with Biblio-MetReS to be able to access the central database. Then, choose Homo sapiens as the organism to work with. There are three types of data sources to choose from, namely, General Engines (Yahoo, Live Search, Altavista, etc.), Literature Database (Medline, Pubmed, Biomed Central, etc.), and Journals (Nature, Science, etc.). Input the DEGs and choose the Literature Database (Medline and Pubmed). Once the search starts, the tool identifies the documents that contain the gene names provided by the user and their synonyms. Then, it extracts the full text from each document, and assesses it for co-occurrence of any pair of genes.

Finally, co-occurrence of any pair of DEGs is extracted, and then, the Cytoscape is used to display the connection between the DEGs. The PATHWAY database records the networks of molecular interactions in the cells, and variants of them specific to particular organisms (http://www.genome.jp/kegg/). The DAVID was used to identify overrepresented pathways by using hypergeometric distribution. The enriched pathway with a corrected p-value (FDR) of $<0.01$ and a count number of $<2$ is considered to be significant.

\section{RESULTS}

The publicly available microarray datasets GSE2378 and GSE13534 were obtained 
from GEO. Using the RankProd packages for the meta-analysis, 13 upregulated genes and 34 downregulated genes with a fold change of $>2$ and a PFP of $<0.05$ were considered to be differentially expressed. At last, 47 DEGs were collected after the meta-analysis.

To procure the relationships between DEGs and other genes, the bibliometric network was constructed using the Biblio-MetReS (Usie et al., 2011) to screen the Medline and Pubmed Database. A total of 24 DEGs and 370 other genes, which are not differentially expressed, displayed in the network (Figure 1). Leucine-rich repeat containing G protein-coupled receptor 5 (LGR5), BCL2/adenovirus E1B $19 \mathrm{kDa}$ interacting protein 3 (BNIP3), interleukin 6 (IL6), enolase 2 (ENO2), thrombomodulin (THBD), and pleiotrophin (PTN) functioned as the hub genes in the network. IL6 connected directly with the fibroblast growth factor 1 (FGF1) and THBD, and indirectly with the Glutathione S-transferase theta 1 (GSTT1), MMP1, and pentraxin 3, long (PTX3). Not only are the hub genes connected to each other, EPHX1 and GSTT1 are connected directly or indirectly through glutathione S-transferase mu 1 (GSTM1), cytochrome P450, family 3 , subfamily A, polypeptide 4 (CYP3A4), and peptidylprolyl isomerase G (cyclophilin G) (PPIG).

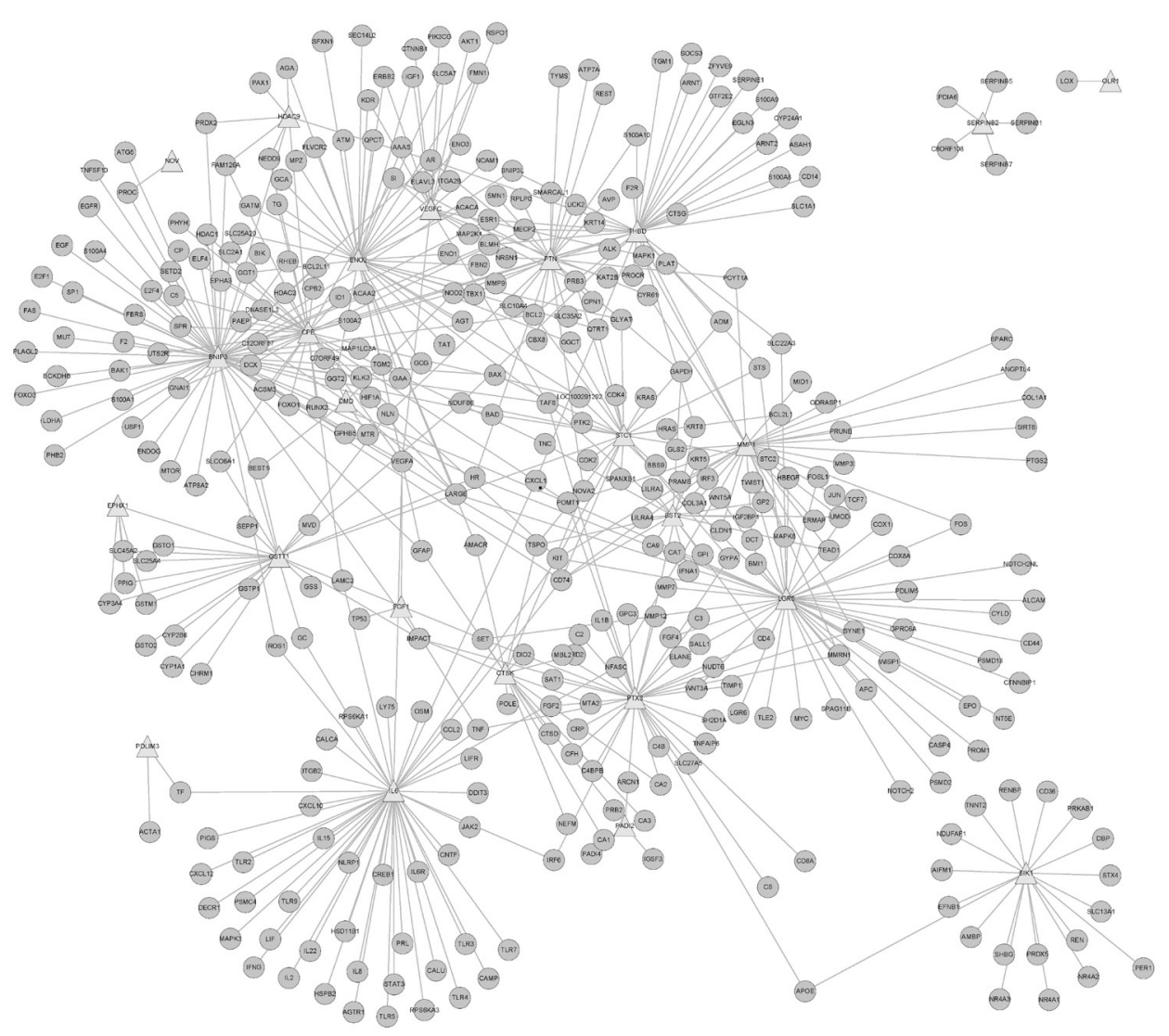

Figure 1. Bibliometric network between differentially expressed genes (DEGs) and other genes. The triangles indicated the DEGs and the ellipses indicated the other genes which were not differentially expressed. 
To assess the significance of the network, we used the overrepresented pathways (also called pathway enrichment analysis) to identify the function of the network. To identify the relevant pathways that are altered in glaucoma, the pathway with an FDR of $<0.05$ and a count of $>2$ was marked as significant pathway by using the DAVID (Huang da et al., 2009).

Quite a few cancer-related pathways were identified (Table 1). Among them, Toll-like receptor signaling pathway, glioma-related pathway, and ErbB signaling pathway were considered as significant pathways based on the findings of enrichment analysis.

\begin{tabular}{|c|c|c|c|c|}
\hline Pathway & Description & Count & $\mathrm{P}$ & FDR \\
\hline hsa05200 & Pathways in cancer & 56 & $1.83 \mathrm{E}-18$ & $2.20 \mathrm{E}-15$ \\
\hline hsa05215 & Prostate cancer & 24 & 4.37E-12 & $5.25 \mathrm{E}-09$ \\
\hline hsa05219 & Bladder cancer & 17 & $1.30 \mathrm{E}-11$ & $1.57 \mathrm{E}-08$ \\
\hline hsa05213 & Endometrial cancer & 17 & $5.38 \mathrm{E}-10$ & $6.46 \mathrm{E}-07$ \\
\hline hsa04620 & Toll-like receptor signaling pathway & 22 & $3.06 \mathrm{E}-09$ & $3.68 \mathrm{E}-06$ \\
\hline hsa05212 & Pancreatic cancer & 18 & $1.38 \mathrm{E}-08$ & $1.66 \mathrm{E}-05$ \\
\hline hsa05218 & Melanoma & 17 & $7.60 \mathrm{E}-08$ & $9.13 \mathrm{E}-05$ \\
\hline hsa05223 & Non-small cell lung cancer & 15 & $7.70 \mathrm{E}-08$ & $9.25 \mathrm{E}-05$ \\
\hline hsa05210 & Colorectal cancer & 18 & $1.59 \mathrm{E}-07$ & $1.90 \mathrm{E}-04$ \\
\hline hsa04012 & ErbB signaling pathway & 17 & $1.46 \mathrm{E}-06$ & 0.001748 \\
\hline hsa05211 & Renal cell carcinoma & 15 & $2.38 \mathrm{E}-06$ & 0.002853 \\
\hline hsa05214 & Glioma & 14 & $3.81 \mathrm{E}-06$ & 0.004574 \\
\hline hsa05220 & Chronic myeloid leukemia & 15 & $5.61 \mathrm{E}-06$ & 0.006742 \\
\hline hsa05221 & Acute myeloid leukemia & 13 & $8.82 \mathrm{E}-06$ & 0.010589 \\
\hline hsa04610 & Complement and coagulation cascades & 14 & $1.10 \mathrm{E}-05$ & 0.013208 \\
\hline hsa04150 & mTOR signaling pathway & 12 & $1.68 \mathrm{E}-05$ & 0.020232 \\
\hline hsa05216 & Thyroid cancer & 9 & $3.20 \mathrm{E}-05$ & 0.038466 \\
\hline hsa04510 & Focal adhesion & 24 & $3.68 \mathrm{E}-05$ & 0.044171 \\
\hline
\end{tabular}

\section{DISCUSSION}

Meta-analysis has proved to be a powerful tool for analyzing microarray experiments by combining data from multiple studies (Hong et al., 2006). Based on this strategy, our analysis has focused on the significant DEGs to reveal the transcriptional responses of glaucoma samples. Our analysis indicated that 47 DEGs have been identified and 24 of them were used to construct bibliometric network with other related genes, including GSTT1, ENO2, carboxypeptidase E (CPE), PTN, prostaglandin D2 synthase $21 \mathrm{kDa}$ (PTGDS), IL6, MMP1, and epidermal growth factor receptor (EGFR).

The glutathione S-transferases (GSTs) are dimeric, cytosolic enzymes that could catalyze the conjugation of multiple toxic endogenous and xenobiotic substrates with reduced glutathione. GSTs bind toxins, function as transport proteins, detoxify endogenous compounds such as peroxidized lipids, and inactivate endogenous end products formed as secondary metabolites during oxidative stress. Therefore, the GST pathway is considered one of the major mechanisms of neutralizing ROS in stressed cells. GSTT1 has been shown to be polymorphically distributed. Studies have shown that the GSTT1*0 allele corresponds to a total or partial deletion of the gene, causing deficiency in enzymatic activity and GSTT1 null genotype may be associated with increased risk of development of POAG in the Turkish (Unal et al., 2007), Egyptian (Rasool et al., 2010), Arab (Abu-Amero et al., 2009), and Pakistani populations.

ENO2 encodes one of the three enolase isoenzymes found in mammals, and this isoenzyme is found in mature neurons and in cells of neuronal origin. Therefore, it is also known 
as neuron-specific enolase (NSE). Recently, several studies have found that approximately $25 \%$ of POAG patients possess serum antibody against NSE and that maximum IOP levels in POAG patients carrying anti-NSE antibody are statistically lower than those in POAG patients not carrying the antibody. During the clinical course, the rates of presence of anti-NSE antibody are significantly higher during the early stages of POAG with visual field deterioration compared to that without it. These observations suggest that the presence of serum autoantibody against NSE may be clinically useful for predicting the progression of visual field loss in POAG patients (Ikeda et al., 2002; Maruyama et al., 2002).

The neuropeptides released by the ciliary epithelium (CE) in the aqueous humor (AH) can serve as messengers to communicate with surrounding tissues in the anterior segment of the eye. In particular, the neuropeptides released by the CE could exert communication between the inflow and outflow pathways and regulate functions, including contractility of the ciliary muscle and outflow resistance of the AH fluid. Alterations to these physiological responses are potentially important links associated with the pathophysiology of glaucoma. However, mature neuropeptides need to be processed by CPE from pro-neuropeptide. Therefore, CPE may be indirectly involved in the excavation of $\mathrm{ONH}$ and visual field loss, and it may eventually cause irreversible blindness (Coca-Prados and Escribano, 2007).

PTN is an $18-\mathrm{kDa}$, heparin-binding, secretory protein that functions to induce mitogenesis, angiogenesis, differentiation, and transformation in vitro. PTN mRNA levels are found to be upregulated in the reactive astrocytes. However, the expression of Ptn gene is significantly lower in the cortical neurons 6 and $24 \mathrm{~h}$ after injury and is undetectable in the degenerating neurons at day 3 . The absence of PTN in injured neurons may contribute to the failure of neurons to survive (Yeh et al., 1998).

PTGDS, also known as glutathione-independent prostaglandin D synthase, catalyzes the conversion of prostaglandin $\mathrm{H} 2$ (PGH2) to prostaglandin D2 (PGD2), a prostaglandin involved in smooth muscle contraction/relaxation and a variety of central nervous system (CNS) functions such as IOP regulation. PTGDS is detected at significantly lower levels in AH of primary congenital glaucoma. Decreased levels of PTGDS in AH may affect the transport of retinoic acid, and consequently the development of the anterior segment structures such as the angle (Bouhenni et al., 2011).

In addition, our results showed that some of these genes might play important roles in glaucoma through cancer-related pathways, including pathways in cancers like prostate cancer, bladder cancer, etc. Of them, the Toll-like receptor signaling pathway, ErbB signaling pathway, and glioma-related pathway were the three significant pathways.

MMP genes were enriched in pathway in cancer. MMPs are important not only for ECM turnover, but also for the interactions between cells and their surroundings. MMP1 expression has been markedly higher in the reactive astrocytes in $\mathrm{ONH}$ with experimental glaucoma (Agapova et al., 2003). Recently, several single-nucleotide polymorphisms (SNPs) for MMP-encoding genes have been reported in POAG patients. A statistically significant increase in the occurrence of $2 \mathrm{G} / 2 \mathrm{G}$ genotype as well as in $2 \mathrm{G}$ allele frequency of MMP1 could be observed in POAG patients compared to healthy controls (Majsterek et al., 2011).

IL-6, Toll-like receptor 4, and Toll-like receptor 2 were enriched in Toll-like receptor signaling pathway. Altered IL-6 has been implicated in the ONH response to early elevated IOP-induced injury. IL- 6 can be produced by all major cellular components of the ONH and exert axonal neuroprotective, regenerative, and proliferative responses. IL-6 can induce astro- 
cytic differentiation from precursor cells. In addition, IL-6 may act on ONH vascular components because IL-6 can stimulate smooth muscle cell proliferation (Johnson et al., 2011). IL-6 levels are significantly higher in the AH of POAG patients compared to the group of patients with cataract (Ghanem et al., 2010). In addition, Sappington et al. (2006) report that pressureconditioned medium from astrocytes reduces RGC survival by another $38 \%$ because of the decreased IL-6 level in the astrocyte medium.

Toll-like receptor proteins are a family of phylogenetically conserved receptors that recognize both self and nonself molecules, and play an important role in both innate and adaptive immunity. Recognition of pathogen-associated molecular patterns by Toll-like receptors activates signaling events that induce the expression of effector molecules such as cytokines and chemokines, which control the adaptive immune responses. Toll-like receptor 4 could interact with exogenous ligands as well as endogenous heat shock proteins (HSPs) such as HSP60, HSP70, and HSP96, but Toll-like receptor2 only recognizes endogenous HSPs, which are noted to show increased expression in the $\mathrm{ONH}$ and as potential candidates for glaucoma antigens (Nakamura et al., 2009). Multiple SNPs in the Toll-like receptor 4 gene are associated with the risk of glaucoma development. This finding suggests that the ligands and/or cytokines involved in the Toll-like receptor 4 signaling network may be risk factors for development of glaucoma (Shibuya et al., 2008).

EGFR was enriched in ErBb pathway and glioma-related pathway. Screening the regulatory pathways of reactive astrocytes, EGFR and ErBb pathways have been shown to have an upstream signal of astrocytes in response to elevated hydrostatic pressure and causes optic nerve astrocytes to express NO synthase and cyclooxygenase-2, change shape, express increased levels of glial fibrillary acidic protein (GFAP), modify the ECM, and proliferate. EGFR (HER, c-erbB1) belongs to a family of transmembrane receptors with intrinsic tyrosine kinase activity. Rapid upregulation of EGFR and its ligands occurs in the astrocytes of optic nerves in patients with glaucoma. Approximately one-third of glioblastomas have EGFR amplifications (Hayashi et al., 2004). A recent study shows that administration of EGFR tyrosine kinase inhibitor promotes significant regeneration of injured optic nerve fibers and prevents the loss of RGCs in a model of glaucomatous optic neuropathy (Liu et al., 2006).

In conclusion, we have used meta-analysis to analyze the whole genome transcription profile of LC cells and astrocytes. Our analysis indicated several DEGs might play crucial roles in the development of glaucoma, including GSTT1, ENO2, CPE, PTN, PTGDS, IL6, MMP1, and EGFR. Further, our results predicted these genes might be involved in glaucoma development through Toll-like receptor signaling pathway, ErbB signaling pathway, glioma and other cancer-related pathways. We anticipate numerous advances in glaucoma research in the coming years based on our meta-analysis findings.

\section{Conflicts of interest}

This has not been published previously elsewhere. The authors report no conflicts of interest. The authors alone are responsible for the content and writing of the manuscript.

\section{ACKNOWLEDGMENTS}

Research supported by the National Natural Science Foundation of China (\#81271726); the Research and Innovation Project of the Shanghai Municipal Education Commission (\# 
12ZZ101); the Pudong New Area Science and Technology Development Fund innovative capital projects (\#PKJ2011-Y320); the Shanghai Jiaotong University Biomedical Engineering (PolyU) Cross Research Fund Project (\#YG2012MS35).

\section{REFERENCES}

Abu-Amero KK, Milcarek B and Bosley TM (2009). GSTM1 and GSTT1 deletion genotypes in various spontaneous optic neuropathies in Arabs. Br. J. Ophthalmol. 93: 1101-1104.

Agapova OA, Kaufman PL, Lucarelli MJ, Gabelt BT, et al. (2003). Differential expression of matrix metalloproteinases in monkey eyes with experimental glaucoma or optic nerve transection. Brain Res. 967: 132-143.

Bouhenni RA, Al SS, Morales J, Wakim BT, et al. (2011). Identification of differentially expressed proteins in the aqueous humor of primary congenital glaucoma. Exp. Eye Res. 92: 67-75.

Chen H and Weber AJ (2001). BDNF enhances retinal ganglion cell survival in cats with optic nerve damage. Invest. Ophthalmol. Vis. Sci. 42: 966-974.

Coca-Prados M and Escribano J (2007). New perspectives in aqueous humor secretion and in glaucoma: the ciliary body as a multifunctional neuroendocrine gland. Prog. Retin. Eye Res. 26: 239-262.

Diboun I, Wernisch L, Orengo CA and Koltzenburg M (2006). Microarray analysis after RNA amplification can detect pronounced differences in gene expression using limma. BMC Genomics 7: 252.

Fuchshofer R (2011). The pathogenic role of transforming growth factor-beta2 in glaucomatous damage to the optic nerve head. Exp. Eye Res. 93: 165-169.

Gentleman RC, Carey VJ, Bates DM, Bolstad B, et al. (2004). Bioconductor: open software development for computational biology and bioinformatics. Genome Biol. 5: R80.

Ghanem AA, Arafa L and Elewa A (2010). Tumor Necrosis Factor- $\alpha$ and Interleukin-6 Levels in Patients with Primary Open-Angle Glaucoma. J. Clin. Exp. Ophthalmol. 2: 1-5.

Hayashi Y, Yamashita J and Watanabe T (2004). Molecular genetic analysis of deep-seated glioblastomas. Cancer Genet. Cytogenet. 153: 64-68.

Hernandez MR (1992). Ultrastructural immunocytochemical analysis of elastin in the human lamina cribrosa. Changes in elastic fibers in primary open-angle glaucoma. Invest. Ophthalmol. Visual Sci. 33: 2891.

Hernandez MR, Agapova OA, Yang P, Salvador-Silva M, et al. (2002). Differential gene expression in astrocytes from human normal and glaucomatous optic nerve head analyzed by cDNA microarray. Glia 38: 45-64.

Hong F, Breitling R, McEntee CW, Wittner BS, et al. (2006). RankProd: a bioconductor package for detecting differentially expressed genes in meta-analysis. Bioinformatics 22: 2825-2827.

Ikeda Y, Maruyama I, Nakazawa M and Ohguro H (2002). Clinical significance of serum antibody against neuron-specific enolase in glaucoma patients. Jpn. J. Ophthalmol. 46: 13-17.

Iwabe S, Moreno-Mendoza NA, Trigo-Tavera F, Crowder C, et al. (2007). Retrograde axonal transport obstruction of brain-derived neurotrophic factor (BDNF) and its TrkB receptor in the retina and optic nerve of American Cocker Spaniel dogs with spontaneous glaucoma. Vet Ophthalmol. 10 (Suppl 1): 12-19.

Johnson EC, Doser TA, Cepurna WO, Dyck JA, et al. (2011). Cell proliferation and interleukin-6-type cytokine signaling are implicated by gene expression responses in early optic nerve head injury in rat glaucoma. Invest. Ophthalmol. Vis. Sci. 52: 504-518.

Kirwan RP, Wordinger RJ, Clark AF and O'Brien CJ (2009). Differential global and extra-cellular matrix focused gene expression patterns between normal and glaucomatous human lamina cribrosa cells. Mol. Vis. 15: 76-88.

Kompass KS, Agapova OA, Li W, Kaufman PL, et al. (2008). Bioinformatic and statistical analysis of the optic nerve head in a primate model of ocular hypertension. BMC Neurosci 9: 93.

Lambert W, Agarwal R, Howe W, Clark AF, et al. (2001). Neurotrophin and neurotrophin receptor expression by cells of the human lamina cribrosa. Invest. Ophthalmol. Vis. Sci. 42: 2315-2323.

Liu B, Chen H, Johns TG and Neufeld AH (2006). Epidermal growth factor receptor activation: an upstream signal for transition of quiescent astrocytes into reactive astrocytes after neural injury. J. Neurosci. 26: 7532-7540.

Majsterek I, Markiewicz L, Przybylowska K, Gacek M, et al. (2011). Association of MMP1-1607 1G/2G and TIMP1 372 $\mathrm{T} / \mathrm{C}$ gene polymorphisms with risk of primary open angle glaucoma in a Polish population. Med. Sci. Monit. 17: CR417-CR421.

Maruyama I, Ikeda Y, Nakazawa M and Ohguro H (2002). Clinical roles of serum autoantibody against neuron-specific enolase in glaucoma patients. Tohoku J. Exp. Med. 197: 125-132.

Miao H, Crabb AW, Hernandez MR and Lukas TJ (2010). Modulation of factors affecting optic nerve head astrocyte migration. Invest Ophthalmol. Vis. Sci. 51: 4096-4103. 
Naka M, Kanamori A, Negi A and Nakamura M (2010). Reduced expression of aquaporin-9 in rat optic nerve head and retina following elevated intraocular pressure. Invest. Ophthalmol. Vis. Sci. 51: 4618-4626.

Nakamura J, Meguro A, Ota M, Nomura E, et al. (2009). Association of toll-like receptor 2 gene polymorphisms with normal tension glaucoma. Mol. Vis. 15: 2905-2910.

Neumann C, Yu A, Welge-Lussen U, Lutjen-Drecoll E, et al. (2008). The effect of TGF-beta2 on elastin, type VI collagen, and components of the proteolytic degradation system in human optic nerve astrocytes. Invest. Ophthalmol. Vis. Sci. 49: 1464-1472.

Pease ME, Zack DJ, Berlinicke C, Bloom K, et al. (2009). Effect of CNTF on retinal ganglion cell survival in experimental glaucoma. Invest. Ophthalmol. Vis. Sci. 50: 2194-2200.

Pena JD, Agapova O, Gabelt BT, Levin LA, et al. (2001). Increased elastin expression in astrocytes of the lamina cribrosa in response to elevated intraocular pressure. Invest. Ophthalmol. Vis. Sci. 42: 2303-2314.

Quigley HA and Broman AT (2006). The number of people with glaucoma worldwide in 2010 and 2020. Br. J. Ophthalmol. 90: 262-267.

Rasool HAA, Nowier SR, Gheith M, Saif ATS, et al. (2010). The risk of primary open angle glaucoma and glutathione S-transferase M1 and T1 polymorphism among Egyptians. J. Am. Sci. 6.

Sappington RM, Chan M and Calkins DJ (2006). Interleukin-6 protects retinal ganglion cells from pressure-induced death. Invest. Ophthalmol. Vis. Sci. 47: 2932-2942.

Shibuya E, Meguro A, Ota M, Kashiwagi K, et al. (2008). Association of Toll-like receptor 4 gene polymorphisms with normal tension glaucoma. Invest. Ophthalmol. Vis. Sci.49: 4453-4457.

Unal M, Guven M, Devranoglu K, Ozaydin A, et al. (2007). Glutathione S transferase M1 and T1 genetic polymorphisms are related to the risk of primary open-angle glaucoma: a study in a Turkish population. Br. J. Ophthalmol. 91: 527530.

Usie A, Karathia H, Teixido I, Valls J, et al. (2011). Biblio-MetReS: a bibliometric network reconstruction application and server. BMC Bioinformatics 12: 387.

Wein FB and Levin LA (2002). Current understanding of neuroprotection in glaucoma. Curr. Opin. Ophthalmol. 13: 61-67.

Yan X, Tezel G, Wax MB and Edward DP (2000). Matrix metalloproteinases and tumor necrosis factor alpha in glaucomatous optic nerve head. Arch. Ophthalmol. 118: 666-673.

Yeh HJ, He YY, Xu J, Hsu CY, et al. (1998). Upregulation of pleiotrophin gene expression in developing microvasculature, macrophages, and astrocytes after acute ischemic brain injury. J. Neurosci. 18: 3699-3707.

Zode GS, Clark AF and Wordinger RJ (2009). Bone morphogenetic protein 4 inhibits TGF-beta2 stimulation of extracellular matrix proteins in optic nerve head cells: role of gremlin in ECM modulation. Glia 57: 755-766.

Zode GS, Sethi A, Brun-Zinkernagel AM, Chang IF, et al. (2011). Transforming growth factor-beta2 increases extracellular matrix proteins in optic nerve head cells via activation of the Smad signaling pathway. Mol. Vis. 17: 1745-1758. 